\author{
(с) Е.А. Трошина, Г.А. Мельниченко, Е.С. Сенюшкина*, Н.Г. Мокрышева
}

«Национальный медицинский исследовательский центр эндокринологии» Минздрава России, Москва, Россия

«Новая болезнь» COVID-19, вызывающая острый респираторный дистресс-синдром, к моменту написания этой статьи поразила уже 5 млн 400 тыс. человек на Земле и унесла жизнь не менее чем 400 тыс. человек более чем в 200 странах. Заболевание может быть как асимптомным, так и протекать с развитием тяжелой вирусной пневмонии, осложниться острым респираторным синдромом и сепсисом, миокардитом и почечной недостаточностью. Хотя вероятность более тяжелого течения отмечена у лиц с сахарным диабетом, и к этим больным в основном привлечено внимание эндокринологов в пандемию, собственно эндокринные проявления COVID-19 пока еще детально не рассматриваются. Данные о поражении коронавирусом гипофиза, гипоталамуса, щитовидной железы (ЩЖ) и надпочечников, их структурных и функциональных нарушениях при инфекции COVID-19 немногочисленны ввиду отсутствия убедительных результатов доклинических и клинических исследований.

Представляемый в этой статье обзор литературы не претендует на роль полноценного систематизированного обзора не только по той причине, что на сегодняшний день в базах данных по ключевым словам «thyroid and COVID-19» и «adrenal and COVID-19», после исключения дублирующих, на момент написания найдена всего 51 работа, но и потому, что эти сообщения сами по себе содержат пока еще мало информации и в основном построены на аналогиях с ранее имевшими место вирусными инфекциями и их роли в развитии патологии оси гипоталамус-гипофиз-надпочечники и гипоталамус-гипофиз-ЩЖ.

КЛЮЧЕВЫЕ СЛОВА: COVID-19; ось гипоталамус-гипофиз-надпочечники; ось гипоталамус-гипофиз-щитовидная железа.

\title{
ADAPTATION OF THE HYPOTHALAMO-PITUITARY-THYROID AND HYPOTHALAMO-PITUITARY- ADRENAL SYSTEMS TO A NEW INFECTIOUS DISEASE - COVID-19 IN THE DEVELOPMENT OF COVID-19 PNEUMONIA AND/OR CYTOKINE STORM
}

\author{
(c) Ekaterina A. Troshina, Galina A. Melnichenko, Evgeniya S. Senyushkina*, Natalia G. Mokrysheva
}

Endocrinology Research Centre, Moscow, Russia

The "new disease" COVID-19, which causes acute respiratory distress syndrome, by the time of writing this article had already affected 5 million 400 thousand people on Earth and claimed the lives of at least 400 thousand people in more than 200 countries. The disease can be either asymptomatic or occur with the development of severe viral pneumonia, complicated by acute respiratory syndrome and sepsis, myocarditis and renal failure. Although the likelihood of a more severe course is noted in individuals with diabetes, and these patients are mainly drawn to the attention of endocrinologists in the pandemic, the actual endocrine manifestations of COVID-19 are not yet considered in detail. There are few data on coronavirus damage to the pituitary, hypothalamus, thyroid gland and adrenal glands, their structural and functional disorders in case of COVID-19 infection, due to the lack of convincing results of preclinical and clinical studies.

The literature review presented in this article does not claim to be a full-fledged systematic review, not only for the reason that to date, only 51 works have been found at the time of writing in the databases for the keywords «thyroid and COVID-19» and "adrenal and COVID-19», after excluding duplicates, but also because these messages themselves contain little information and are mainly based on analogies with previously existing viral infections and their role in the development of hypothalamus-pituitary axis pathology-adrenal glands and hypothalamus-pituitary-thyroid.

KEYWORDS: COVID-19; axis hypothalamo-pituitary-adrenals; axis hypothalamo-pituitary-thyroid. 


\section{КОНСЕНСУСЫ СОВЕТА ЭКСПЕРТОВ}

Международные и национальные ассоциации подготовили рекомендации по ведению пациентов с различными эндокринопатиями (от сахарного диабета до гипер- и гипокортицизма) в период пандемии COVID-19 [1], но они скорее направлены на поддержку принятия клинически взвешенных решений в коморбидных ситуациях при ограниченности врачебных ресурсов, чем на оценку собственно проявлений эндокринопатий при COVID-19.

Рассматривая вопрос о тиреоидной патологии и COVID-19, Британская ассоциация тиреоидологов, Британская ассоциация эндокринологов [2], как и Российская ассоциация эндокринологов [3], подготовили консенсусы о ведении пациентов с тиреоидной патологией в период пандемии COVID-19. Эти рекомендации базировались на общих принципах ведения лиц с тиреотоксикозом и гипотиреозом в условиях вирусных эпидемий, разъяснялась необходимость продолжать ранее назначенную терапию, а также обращалось внимание на сходство симптомов агранулоцитоза и инфекционных болезней и давались разъяснения по их дифференциальной диагностике (прерывание терапии тиреостатиками и исследование общеклинического анализа крови) [4]. Было опубликовано и разъяснение по поводу порядка проведения пункционных биопсий и хирургических методов лечения/радиойодтерапии рака щитовидной железы (ЩЖ) в условиях пандемии [5].

В частности, по заключению Британской ассоциации тиреоидологов, пациентам, получающим заместительную терапию тиреоидными гормонами или тиреостатическую терапию, рекомендуется продолжать принимать препараты в обычном режиме. Однако отмечено, что при развитии агранулоцитоза как побочного эффекта тиреостатической терапии его симптомы часто пересекаются с симптомами COVID-19, что зачастую затрудняет дифференциальную диагностику. В данном случае рекомендуется немедленно прекратить прием препарата и сдать развернутый общеклинический анализ крови в ближайшее время [6].

\section{НОВЫЕ ЗАДАЧИ В НЕПРОСТЫХ УСЛОВИЯХ...}

Во многом сегодняшняя ситуация с данными по состоянию эндокринной системы при новой инфекции объясняется не только новизной проблемы, но и беспрецедентным вовлечением в ликвидацию пандемии врачебных ресурсов и материальных средств. Разумеется, в будущем нас ждут новые интересные данные и новые обзоры, но пока воспользуемся уникальной ситуацией: эпидемия COVID-19, в ликвидации которой приняли участие врачи многих специальностей (в частности, COVID-центр на 127 коек был открыт в ФГБУ «НМИЦ эндокринологии» Минздрава России, и один из авторов статьи (д.м.н., проф., член-корр. РАН Екатерина Анатольевна Трошина) непосредственно работала «в красной зоне», стала во многом загадкой для врачей на момент своего появления.

В особых условиях оказались многие специалисты, не имеющие опыта в проведении респираторной поддержки пациентам с тяжелой острой дыхательной недо- статочностью. Помимо первоочередной задачи спасения жизни больных, в условиях отсутствия доказанных эффективных методов лечения и специфической профилактики, уникальный препарат, оказавшийся эффективным в предупреждении развития цитокинового шторма, Тоцилизумаб, после нескольких лет применения в ревматологии был недавно одобрен FDA для лечения эндокринной офтальмопатии (ЭОП) [7] и также оказался эффективен при лечении пневмонии, вызванной COVID-19.

\section{ПРОГНОЗ ОТДАЛЕННЫХ ПОСЛЕДСТВИЙ}

До настоящего момента не вполне понятно, какие остаточные явления ожидают перенесших в той или иной форме данный воспалительный процесс, в том числе как скажется на состоянии щЖ, надпочечников и гипофиза и сама инфекция, и те методы лечения, которые предпринимались для спасения этих больных; и здесь очевидно, по предыдущему опыту наблюдения за больными с SARS MERS, что нас могут ожидать остаточные функциональные и морфологические повреждения эндокринной системы.

Хорошо известно, что вирусные поражения щЖ рассматриваются чаще всего в контексте триггера подострого тиреоидита, «молчащего тиреоидита», иммуногенного тиреотоксикоза или гипотиреоза [8], при этом прямые доказательства присутствия вируса в тканях получены для ретровируса и вируса паротита при подостром тиреоидите, ретровирусов (HTLV-1, HFV, HIV и SV40) при болезни Грейвса и для HTLV-1, энтеровируса, вирусов краснухи, паротита, HSV, вируса Эпштейна-Барр и парвовируса при тиреоидите Хашимото, но из этого не следует, что именно они отвечают за развитие патологии, равно как и не являются безучастными свидетелями.

Прошедшие в целом незаметно для Европы эпидемии SARS и MERS (атипичные пневмонии, свиной и птичий грипп) были куда более агрессивными, с несравненно большим коэффициентом репродукции и летальности, также было накоплено мало данных о состоянии ЩЖ при SARS, хотя имелись сведения о том, что у существенного числа больных с SARS выявлены аномалии в уровнях тиреоидных гормонов как в острую фазу, так и при выздоровлении, и наиболее простым объяснением было предположение о том, что эти изменения укладываются в рамки sick-euthyroid синдрома (синдрома нетиреоидной болезни, синдрома низкого трийодтиронина $\left(\mathrm{T}_{3}\right)$ ) [9].

Так, в исследовании, проведенном во время вспышки атипичной пневмонии в 2003 г., было показано, что уровни $\mathrm{T}_{3}$ и тироксина $\left(\mathrm{T}_{4}\right)$ в сыворотке были ниже у пациентов с атипичной пневмонией по сравнению с контрольной группой как в острой, так и в реконвалесцентной фазах, что расценивалось как синдром эутиреоидной патологии. Отмечалось снижение средней массы ЩЖ в результате уменьшения размера фолликулов и истощения коллоида [10]. При исследовании аутопсийного материала у 5 пациентов было выявлено заметное разрушение фолликулярных и парафолликулярных клеток щЖ [11]. Разрушение фолликулярных клеток сопровождается снижением $\mathrm{T}_{3}$ и $\mathrm{T}_{4}$; в то же время повреждение парафолликулярных клеток теоретически может привести к низким уровням сывороточного кальцитонина. Последнее утверждение предложено в качестве 
вероятного механизма остеонекроза головки бедренной кости, наблюдаемого у выздоровевших пациентов с ОРВИ. Дефицит кальцитонина приводит к расторможению остеокластов и, как следствие, к остеонекрозу.

Известно, что при системных заболеваниях может развиваться синдром низкого $\mathrm{T}_{3}$ [12]. Поэтому вполне ожидаемо, что тяжелое течение COVID-19 будет сопровождаться таким отклонением, особенно если у пациента имеется лихорадка и поражаются нижние отделы дыхательных путей. Таким образом, не рекомендуется исследование функции ЩЖ в остром периоде COVID-19.

На сегодня есть только одна публикация A. Brancatella и соавт. (2020) [13] о развитии у 18-летней девушки яркой клинической картины, типичной для подострого тиреоидита, через 15 дней после SARS-CoV-2-позитивного-орофарингеального мазка, взятого по поводу симптоматики нетяжелого COVID-19, от которого девушка выздоровела в течение нескольких дней. Помимо типичной клинической картины, факт ТТГ-независимого тиреотоксикоза был подтвержден высоким уровнем св. $\mathrm{T}_{4}$ и $\mathrm{T}_{3}$, подавленным ТТГ, типичными маркерами воспаления и лейкоцитозом, имелось гипоэхогенное диффузное двустороннее поражение ЩЖ при ультразвуковом исследовании. Преднизон дал отчетливый эффект через 48 ч (положительный тест Крайля). Функция ЩЖ восстановилась, а маркеры воспаления нормализовались через 1,5 месяца.

\section{COVID-19 И ДИСФУНКЦИЯ ЩИТОВИДНОЙ ЖЕЛЕЗЫ}

Ключевую роль в развитии заболеваний ЩЖ имеют нарушения в функционировании гипоталамо-гипофизарно-тиреоидной системы, в том числе и нарушения в биосинтезе гормонов ЩЖ, связанные с различными средовыми и/или генетическими факторами. Патология ЩЖ многогранна благодаря своим этиопатологическим механизмам.

Аутоиммунные заболевания ЩЖ (АИЗЩЖ) - это в первую очередь нарушение иммунорегуляции в сочетании с органической дисфункцией, являющейся следствием антигенспецифической атаки, дополняемой недостаточной супрессией (и, следовательно, активацией) лимфоцитов, действие которых направлено на антигены на определенных клетках-мишенях, т. е. тироцитах, а также в сочетании с выработкой различных цитокинов (например, интерферона гамма (ИФН-ү)), воздействующих на клетки-мишени с близкого расстояния. Нарушение иммунологической толерантности лежит в основе формирования АИЗЩЖ, в т. ч. в составе аутоиммунных полигландулярных синдромов [14].

Вирусный или микробный антиген, обладающий сходством с аутоантигеном (молекулярная мимикрия), способен запускать выработку аутоантител, которые вступают в перекрестную реакцию с аутоантигеном, после чего иммунный ответ вступает в реакцию с соответствующими структурами аутологичных клеток. Многими исследователями высказываются предположения, что АИЗЩЖ «вероятно, провоцируется каким-то внешним фактором, например, инфекцией, и этот фактор запускает экспрессию тироцитами HLA-DR, которая и приводит к их развитию», тем не менее сторонники данной теории признают и дополнительную необходимость в наруше- нии функционирования иммунной системы. Тиреоидные клетки могут вследствие цитокиновой стимуляции или комплементной атаки продуцировать некоторые другие иммуноактивные молекулы (например, простагландин-Е2, ИЛ-6 и ИЛ-8), что дополнительно усиливает тироцитно-иммуноцитную сигнализацию [15].

В данное время отсутствуют какие-либо доказательства, свидетельствующие о том, что пациенты, имеющие АИЗЩЖ, более подвержены вирусным инфекциям, в т. ч. и SARS-CoV-2, и имеют более тяжелое течение COVID-19. Отдельные группы пациентов, такие как пациенты с ЭОП, получающие иммуносупрессивную терапию, возможно, имеют повышенный риск развития тяжелой коронавирусной инфекции [2].

Отмечено, что тиреостатики не увеличивают риск развития вирусной инфекции, в т. ч. и COVID-19; так же, как и отсутствуют данные о тяжелом течении коронавирусной инфекции на фоне тиреостатической терапии [2].

Нет убедительных данных в отношении увеличения частоты заболевания COVID-19 среди пациентов с неконтролируемой функцией ЩЖ. Однако предполагается, что пациенты с тиреотоксикозом могут быть подвержены более высокому риску осложнений инфекционных заболеваний [16]. В данном случае для снижения таких рисков необходим регулярный прием тиреостатиков. В ряде исследований у пациентов с аутоиммунным тиреоидитом в высоких концентрациях был обнаружен цитокин - ИЛ-6, уровень которого коррелировал с увеличением количества других Т-хелперов - Th22, которые, в свою очередь, были ассоциированы с уровнем антител к тиреопероксидазе [17].

Есть единичные наблюдения увеличения потребности в тироксине после выздоровления от нетяжелой формы COVID-19 (д.м.н., проф., академик РАН Галина Афанасьевна Мельниченко, дистанционное консультирование).

Отсутствуют доказательства в отношении повышения риска развития вирусной инфекции, включая COVID-19, у пациентов после радиойодтерапии или операции на ЩЖ [2].

\section{СТРУКТУРНОЕ ПОВРЕЖДЕНИЕ ЩИТОВИДНОЙ ЖЕЛЕЗЫ SARS}

Lan Weia, и соавт. (2003) [18] пытались найти любое потенциально возможное повреждение собственно ткани ЩЖ, вызванное SARS, в образцах, полученных при аутопсии 5 больных в сравнении с 10 пациентами, умершими от других причин с неповрежденной ЩЖ.

Поскольку сверхэкспрессия некоторых неструктурированных белков SARS-CoV, как было показано ранее, способна индуцировать апоптоз, эти исследователи предположили, что не функциональные нарушения, ведущие к синдрому нетиреоидальной патологии, а непосредственное поражение железы приводит к снижению продукции тиреоидальных гормонов.

Метод терминальной дезоксинуклеотидилтрансфераз-опосредованной реакции dUTP-метки 3'-гидроксильного конца (terminal deoxynucleotidyl transferasemediated dUTP nick end-labeling, TUNEL) был использован для идентификации клеточного апоптоза, и было показано, что фолликулярный эпителий повреждался и слоями 
перемещался внутрь фолликула. Данный метод выявлял множество подвергшихся апоптозу клеток, структура самого фолликула была деформирована: фолликулы становились расширенными или, наоборот, сплющенными, отсутствовали и кальцитонин-позитивные клетки. То есть как парафолликулярный, так и фолликулярный аппараты были повреждены, и апоптоз становился наиболее очевидной причиной снижения уровней гормонов. Однако сохраняющееся при этом снижение ТТГ заставляет думать о том, что первично поражалась не только щЖ у данных больных.

\section{ПАТОГЕННОСТЬ ВИРУСА И ОЦЕНКА РИСКОВ}

В настоящее время известно, что ангиотензинпревращающий фермент 2 (АПФ2) человека является рецептором и «точкой входа» в клетку некоторых коронавирусов. Он экспрессируется в большинстве тканей, в т. ч. и в ряде органов эндокринной системы, таких как поджелудочная железа, ЩЖ, яички, яичники, надпочечники и гипофиз $[19,20]$.

R. Pal и M. Banerjee (2020) [6] подчеркивают, что на сегодняшний день недостаточно знаний о возможных поражениях эндокринной системы у пациентов с COVID-19. Например, известно, что АПФ2 выполняет роль рецептора для коронавируса в пневмоците, но, в свою очередь, РНК вируса определяется в плазме, что подтверждает тот факт, что вирус может взаимодействовать с АПФ2 и в других тканях [21]. Для предположения того, что эндокринная система восстановится без последствий после взаимодействия с SARS-CoV-2 через АПФ2-рецепторы, экспрессированные на ее клетках, пока нет ни клинических, ни преклинических данных.

Накопление данных о полиморфных вариантах гена, кодирующего АПФ2, его метилировании или гиперэкспрессии на поверхности Т-клеток, деметилировании/ метилировании генов, регулирующих обмен цитокинов и интерферона, а также генов-регуляторов иммунного ответа (например, подозреваемых в развитии аутоиммунных тиреопатий) могло бы стать предметом исследования.

По аналогии с SARS [22] не исключено, что SARS-CoV-2 вызывает гипофизит или поражает гипоталамус за счет отека и дегенерации нейронов, тем более что случаи энцефалита при COVID-19 уже описаны. Вероятно, в ближайшем будущем перспективными станут исследования в отношении пациентов, перенесших COVID-19, по оценке риска повреждения гипоталамуса и гипофиза, следствием которого может стать развитие вторичного (центрального) гипотиреоза [23, 24].

Типичная неврологическая манифестация - нарушение обоняния - может быть объяснена экспрессией АПФ2 на ольфакторных эпителиальных клетках [25]. Ткани гипоталамуса и гипофиза также экспрессируют АПФ2 и теоретически могут стать мишенью для вируса. На аутопсии отек и дегенерация нейронов и идентификация в них SARS-генома были продемонстрированы в гипоталамусе. Биохимические доказательства вовлечения гипоталамо-гипофизарной системы при SARS обнаружены Leow и соавт. в 2005 г. [22]. Пережившие SARS больные (61 участник) были обследованы этой группой через 3 месяца после выздоровления и затем наблюдались периодически. У 40\% был выявлен центральный гипокортицизм, и у 62,5\% из них функция оси гипофиз-надпочечники нормализовалась. Из них 87,5\% предъявляли типичные жалобы на слабость и постуральное головокружение. У 5\% также был центральный гипотиреоз.

Предполагалось развитие у этих больных гипофизита или гипоталамо-гипофизарной дисфункции. Теоретически ее косвенным признаком стала бы регистрация явлений несахарного диабета, но в настоящее время по понятным причинам проблемы гипернатриемии у лиц с тяжелым течением COVID-19 рассматриваются в контексте влияния пирексии, а случаев развития несахарного диабета после выздоровления от COVID-19 не описано. Можно предполагать, что снижение адаптационных резервов за счет гипоталамо-гипофизарной дисфункции (возможно, вследствие энцефалита), гипофизита создаст в клиническом отношении модель сочетания первичного гипотиреоза (транзиторного, деструктивного, в том числе с транзиторной тиреотоксической фазой аутоиммунного) с центральным гипокортицизмом. Их сочетание может объяснять длительный период реконвалесценции и формирование неспецифических эндокринных симптомов и синдромов при выздоровлении, которые имели место при так называемом post-SARSsickness syndrome.

\section{НАДПОЧЕЧНИКОВАЯ НЕДОСТАТОЧНОСТЬ КАК СЛЕДСТВИЕ ПЕРЕНЕСЕННОГО COVID-19}

Высказывается гипотеза, что некоторые аминокислотные последовательности вирусов SARS-CoV, как и вируса гриппа, имеют молекулярное сходство с адренокортикотропный гормон (АКТГ), и так называемая «иммуноинвазивная стратегия» вируса реализуется благодаря этому сходству в снижении выброса кортизола в ответ на стресс в организме подвергшегося инфекционной атаке человека. Кроме того, антитела к вирусу приобретают и способность перекрестно инактивировать АКТГ [26]. Большинство белков SARS-CoV-2 имеют 95-100\% гомологию с белками молекулы SARS-CoV, что позволяет допустить способность SARS-CoV-2 включать те же механизмы молекулярной мимикрии [27], и больной с тяжелой формой COVID-19 может быть в группе риска так называемой «глюкокортикоидной недостаточности критически тяжелого больного».

Ситуация усугубляется для клиницистов тем, что, во-первых, сами диагностические критерии этого синдрома недостаточно проработаны, во-вторых, назначение фармакологических доз глюкокортикоидов при тяжелом течении COVID-19 в первых рекомендациях отвергалось и приветствовалось в последующих [28], следовательно, сложно оценить вклад собственно болезни и подавления большими дозами кортикостероидов надпочечников. До настоящего времени оценка результатов кратковременного назначения высоких доз глюкокортикоидов при тяжелом течении SARS вызывала вопросы, и не рекомендовалось переносить эту методику на всех больных COVID-19.

Известно, что ИЛ-1 и ИЛ-6, вырабатываемые воспалительными клетками, являются стимуляторами эндокринной системы посредством синтеза АКТГ. Данный импульс, по-видимому, проходитпогипоталамическим рецепторам, 
вследствие чего центральная нервная система вступает во взаимодействие с эндокринной и иммунной системами в ответ на воздействие патогенов. Более того, данная связь свидетельствует о том, что регуляция гипоталамо-гипофизарно-надпочечниковой оси цитокинами при воспалении зависит от кортикотропинвысвобождающего гормона. Однако длительная стимуляция с помощью ИЛ-6 не гарантирует устойчивого повышения уровня АКТГ. В действительности, хроническое воспаление у пациентов с АИЗ, по всей видимости, коррелирует с измененной функцией гипоталамо-гипофизарно-надпочечниковой оси, поскольку доказано, что показатели соотношения между сывороточным кортизолом и воспалительными цитокинами (количество ИЛ-6 и фактора некроза опухоли (ФНО) может достигать уровня, в 10 раз превышающего нормальные значения) намного выше у здоровых людей, чем у пациентов с ревматоидным артритом. Предполагается также, что относительная недостаточность надпочечников у таких пациентов может быть обусловлена нарушениями функционирования печени при метаболизме стероидных гормонов. На самом деле стимуляция основного фермента, задействованного в синтезе, $11 \beta$-гидроксистероиддегидрогеназы (11ß-ГСД) 1 типа в значительной степени осуществляется ФНО и другими провоспалительными цитокинами. Преобразование в активизированный гормон происходит в различных орга-

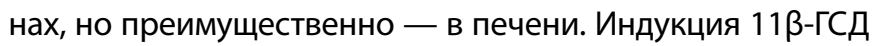
и обусловленное ею увеличение концентрации активных гормонов могут провоцировать отрицательную обратную реакцию и, следовательно, дисфункцию гипоталамо-гипофизарно-надпочечниковой оси.

Как и у других циркулирующих регуляторных молекул, уровень кортизола подчиняется циркадному ритму, достигая пика ранним утром, а нижней точки - поздно вечером. Кортизол регулирует уровни нескольких циркулирующих в крови провоспалительных цитокинов, таких как ИЛ-2, ИЛ-3, ИЛ-6, ФНО-а и ИФН-ү. Кроме того, он влияет на активность и жизнеспособность клеток иммунной системы. Глюкокортикоиды также угнетают фагоцитоз антигенов и их последующую элиминацию макрофагами. Они угнетают как клеточный, так и гуморальный иммунный ответ, поддерживая баланс про- и противовоспалительных реакций, и вызывают инволюцию лимфоидных органов. Кортизол угнетает фагоцитарную активность нейтрофилов и макрофагов, подавляет активность лимфоцитов, тормозя их созревание и дифференцировку, стимулируя апоптоз. За счет иммуносупрессивного эффекта глюкокортикоиды снижают количество и активность воспалительных клеток, особенно тканевых макрофагов, и ограничивают их способность реагировать на поступающие антигены. Подавление активности иммунных клеток нарушает их дегрануляцию и высвобождение разрушающих ткани ферментов (матриксных металлопротеиназ, протеаз, нуклеаз и др.), хемоаттрактантов, адгезивных молекул [29].

Несмотря на вышеизложенное, при оценке результатов лечения 31 больного с COVID-19 не удалось доказать, что терапия глюкокортикостероидами ускоряет выведение вируса, уменьшает сроки госпитализации или продолжительность симптомов [28]. Продолжается рандомизированное клиническое исследование эффективности и безопасности глюкокортикостероидов при COVID-19 (NCT04273321).
Разумеется, люди с надпочечниковой недостаточностью, первичной или вторичной, а также длительно получавшие глюкокортикостероиды, находятся в группе риска инфекций дыхательных путей и должны продолжать терапию глюкокортикостероидами в период пандемии с увеличением дозы в случае присоединения болезни по общим правилам «дней болезни» этих больных, во избежание адреналового криза.

\section{МОНОКЛОНАЛЬНЫЕ АНТИТЕЛА - АДАПТАЦИЯ К НОВОЙ ТЕРАПИИ}

Еще одной важной составляющей частью адаптации эндокринной системы к перенесенной инфекции COVID-19 может стать адаптация к одному из наиболее эффективных методов ее лечения - использованию моноклональных антител.

К счастью для эндокринологов, моноклональное антитело, эффективно позволяющее блокировать цитокиновый шторм (важное звено возможного фатального прогноза при COVID-19; Тоцилизумаб - моноклональное антитело против ИЛ-6, экспрессирующегося на адипоцитах, фибробластах и макрофагах, что приводит к формированию ЭОП), уже разрешено для лечения офтальмопатии FDA [30, 31], и, таким образом, проводя данную терапию, мы не должны рассматривать ее с позиций негативных влияний на эндокринную систему.

ИЛ-6 представляет собой провоспалительный цитокин, вырабатываемый различными типами клеток, включая Т- и В-лимфоциты, моноциты и фибробласты. Он участвует в различных физиологических процессах, таких как активация Т-клеток, индукция секреции иммуноглобулина, индукция синтеза печеночно-фазовых белков в печени и стимуляция гемопоэза. ИЛ-6 присутствует в высоких концентрациях у пациентов с ЭОП и играет важную роль в патогенезе заболевания. Особое значение В- и Т-лимфоциты имеют на ранних этапах развития ЭОП. Предполагается, что дальнейшее прогрессирование заболевания включает привлечение Т-клеток в орбиту для участия в ответной иммунной активации и усиления В-клеточных ответов, что, в свою очередь, приводит к воспалительным процессам, таким как продуцирование цитокинов (включая ИЛ-6) и простагландинов, в результате чего происходит ремоделирование мягких тканей глазницы, характерное для ЭОП.

Тоцилизумаб является биологическим агентом, который успешно применяется и при других АИЗ, например, при ревматоидном артрите. Некоторые исследования [30] продемонстрировали эффективность Тоцилизумаба при лечении пациентов с активной ЭОП средней и тяжелой степени тяжести, но нет четких рекомендаций относительно его применения [32].

Если, используя так называемые ингибиторы иммунного ответа, мы вынуждены считаться с потенциальными негативными влияниями на эндокринную систему, то при применении Тоцилизумаба мы, скорее, поражены патофизиологическому сходству (участие ИЛ-б) в развитии столь клинически несхожих проявлений, как ЭОП, цитокиновый шторм (по сути гиперергическая реакция иммунной системы), и у нас мало аналогов подобной реакции и данных о ее предикторах. 


\section{ЗАКЛЮЧЕНИЕ}

Заболевания эндокринной системы (сахарный диабет, тиреопатии, аутоиммунные полигландулярные синдромы, надпочечниковая недостаточность, ЭОП и т.д.), В развитии которых важную роль играют нарушения иммунного ответа, относятся к числу наиболее тяжелых хронических болезней человека.

Важнейшим вызовом сегодняшнего дня стала новая инфекция COVID-19. Клинические особенности ее течения, вероятные осложнения, внедряемые и апробируемые схемы лечения, плейотропные и нежелательные эффекты различных лекарственных препаратов, длительность реабилитации пациентов - все это исключительно важно для пациентов с эндокринной патологией, в т. ч. аутоиммунного генеза. Кроме того, существует и очевидная возможность провоцирующего действия COVID-19 на развитие и прогрессирование эндокринопатий.

Изучение механизмов влияния COVID-19 на эндокринную систему в настоящее время находится «на старте». Накопление опыта, его анализ, проведение научных исследований - все это в самой ближайшей перспективе. Результаты таких исследований будут исключительно важны с учетом широкой распространенности и высокой заболеваемости, инвалидизирующих осложнений целого ряда заболеваний органов эндокринной системы. Первые обобщения и гипотезы о возможных механизмах влияния коронавирусной инфекции на эндокринную систему представлены в данном обзоре литературы.

\section{ДОПОЛНИТЕЛЬНАЯ ИНФОРМАЦИЯ}

Источник финансирования. Поисково-аналитическая работа по подготовке рукописи проведена в рамках гос. задания «Эпидемиологические и молекулярно-клеточные характеристики опухолевых, аутоиммунных и йододефицитных тиреопатий как основа профилактики осложнений и персонализации лечения».

Конфликт интересов. Авторы декларируют отсутствие явных и потенциальных конфликтов интересов, связанных с публикацией настоящей статьи.

Участие авторов: Е.А. Трошина, Г.А. Мельниченко, Е.С. Сенюшкина - сбор и анализ данных литературы, написание основного текста статьи; Н.Г. Мокрышева - редактирование текста статьи. Все авторы внесли значимый вклад в проведение поисково-аналитической работы и подготовку статьи, прочли и одобрили финальную версию до публикации.

\section{СПИСОК ЛИТЕРАТУРЫ | REFERENCES}

1. Kaiser UB, Mirmira RG, Stewart PM. Our response to COVID-19 as endocrinologists and diabetologists. J Clin Endocr Metab. 2020;105(5):dgaa148. Doi: 10.1210/clinem/dgaa148.

2. Boelaert K, Visser WE, Taylor PN, et al. Endocrinology in the time of COVID-19: management of hyper- and hypo-thyroidism. Eur J Endocrinol. 2020;183(1):G33-G39. Doi: 10.1530/EJE-20-0445.

3. Мокрышева Н.Г., Галстян Г.Р., Киржаков М.А., и др. Пандемия COVID-19 и эндокринопатии // Проблемы эндокринологии. 2020. — T.66. — №1. [Mokrysheva NG, Galstyan GR, Kirzhakov MA, et al. Pandemiya COVID-19 i endokrinopatii. Problemy endokrinologii. 2020;66(1). (In Russ.)].

4. Сборник методических рекомендачий, алгоритмов действий медичинских работников на различных этапах оказания помощи, чек-листов и типовых документов, разработанных на период наличия и угрозы дальнейшего распространения новой коронавирусной инфекиии в Санкт-Петербурге. Версия 1,0 от 17.04.2020. — СПб.; 2020. - 157 с. [доступ от 21.03.2012]. [Sbornik metodicheskikh rekomendatsii, algoritmov deistvii meditsinskikh rabotnikov na razlichnykh etapakh okazaniya pomoshchi, chek-listov i tipovykh dokumentov, razrabotannykh na period nalichiya i ugrozy dal'neishego rasprostraneniy a novoi koronavirusnoi infektsii v Sankt-Peterburge. Version 1,0 dated 17.04.2020. St. Petersburg; 2020. 157 p. (In Russ.)]. Доступ по ссылке http://docs.cntd.ru/document/564778217.

5. Vrachimis A, lacovou I, Giannoula E, Giovanella L. Endocrinology in the time of COVID-19: management of thyroid nodules and cancer. Eur J Endocrinol. 2020;183(1):G41-G48. Doi: 10.1530/EJE-20-0269.

6. Pal R, Banerjee M. COVID 19 and the endocrine system: exploring the unexplored. J Endocrinol Invest. 2020;43(7):1027-1031. Doi: 10.1007/s40618-020-01276-8.

7. Perez-Moreiras JV, Gomez-Reino JJ, Maneiro JR, et al. Efficacy of Tocilizumab in patients with moderate-to-severe corticosteroidresistant graves orbitopathy: a randomized clinical trial. Am J Ophthalmol. 2018;195:181-190. Doi: 10.1016/j.ajo.2018.07.038.

8. Desailloud R, Hober D. Viruses and thyroiditis: an update. Virol J. 2009;6:5. Doi: 10.1186/1743-422X-6-5

9. Трошина Е.А., Ванушко В.Э. Заболевания щитовидной железы. Аутоиммунные полигландулярные синдромы. Глава 2. В кн.: Персонализированная эндокринология в клинических примерах. / Под ред. И.И. Дедова. - М.: ГЭОТАР-Медиа; 2018. C. 9-84. [Troshina EA, Vanushko VE. Zabolevaniya shchitovidnoi zhelezy. Autoimmunnye poliglandulyarnye sindromy. Part 2. In:
Personalizirovanaya endokrinologiva $v$ klinicheskikh primerakh Ed by I.I. Dedov. Mossow: GEOTAR-Media; 2018. p. 9-84. (In Russ.)].

10. De Jongh FE, Jobsis AC, Elte JW. Thyroid morphology in lethal non-thyroidal illness: a post-mortem study. Eur J Endocrinol. 2001;144(3):221-226. Doi: 10.1530/eje.0.1440221.

11. Wei L, Sun S, Xu C, et al. Pathology of the thyroid in severe acute respiratory syndrome. Hum Pathol. 2007;38(1):95-102. Doi: 10.1016/j.humpath.2006.06.011.

12. Fliers E, Bianco AC, Langouche L, Boelen A. Thyroid function in critically ill patients. Lancet Diabetes Endocrinol. 2015;3(10):816-825. Doi: 10.1016/S2213-8587(15)00225-9.

13. Brancatella A, Ricci D, Viola N, et al. Subacute thyroiditis after SARS-CoV-2 infection. J Clin Endocrinol Metab. 2020;105(7):dgaa276. Doi: 10.1210/clinem/dgaa276.

14. Здор В.В., Маркелова Е.В., Гельцер Б.И. Новые участники нарушения толерантности к антигенам щитовидной железы: к концепции иммунопатогенеза аутоиммунных заболеваний щитовидной железы // Медииинская иммунология. - 2016. T.18. — №3. — C. 209-220. [ZdorW, Markelova EV, Gel'tser BI. Novye uchastniki narusheniya tolerantnosti $\mathrm{k}$ antigenam shchitovidnoi zhelezy: k kontseptsii immunopatogeneza autoimmunnykh zabolevanii shchitovidnoi zhelezy. Meditsinskaya immunologiya. 2016;18(3):209-220. (In Russ.)].

15. Dong YH, Fu DG. Autoimmune thyroid disease: mechanism, genetics and current knowledge. Eur Rev Med Pharmacol Sc. 2014;18(23):3611-3618

16. De Leo S, Lee SY, Braverman LE. Hyperthyroidism. Lancet. 2016;388(10047):906-918. Doi: 10.1016/S0140-6736(16)00278-6.

17. Nielsen $\mathrm{CH}$, Bendtzen $\mathrm{K}$. Immunoregulation by naturally occurring and disease-associated autoantibodies: binding to cytokines and their role in regulation of T-cell responses. Adv Exp Med Biol. 2012;750:116-132. Doi: 10.1007/978-1-4614-3461-0_9.

18. Wei L, Sun $\mathrm{S}, \mathrm{Xu} \mathrm{CH}$, et al. Pathology of the thyroid in severe acute respiratory syndrome. Hum Pathol. 2007;38(1):95-102. Doi: 10.1016/j.humpath.2006.06.011

19. Liu F, Long X, Zou W, et al. Highly ACE2 expression in pancreas may cause pancreas damage after SARS-CoV-2 infection. medRxiv 2020.02.28.20029181 [Internet] [cited 2020 Apr 1]. Available from: https://www.medrxiv.org/content/10.1101/2020.02.28.20029181v1. Doi: 10.1101/2020.02.28.20029181.

20. Li W, Zhang B, Lu J, et al. The characteristics of household transmission of COVID-19. J Clin Infect Dis. 2020:ciaa450. Doi: 10.1093/cid/ciaa450. 
21. Chang L, Yan Y, Wang L. Coronavirus disease 2019: coronaviruses and blood safety. Transfus Med Rev. 2020;34(2):75-80. Doi: 10.1016/j.tmrv.2020.02.003.

22. Leow MK, Kwek DS, Ng AW, et al. Hypocortisolism in survivors of severe acute respiratory syndrome (SARS). Clin Endocrinol (Oxf). 2005;63(2):197-202. Doi: 10.1111/j.1365-2265.2005.02325.x.

23. Gu J, Gong E, Zhang B, et al. Multiple organ infection and the pathogenesis of SARS. J Exp Med 2005;202(3):415-424. Doi: 10.1084/jem.20050828.

24. Wei L, Sun S, Zhang J, et al. Endocrine cells of the adenohypophysis in severe acute respiratory syndrome (SARS). Biochem Cell Biol. 2010;88(4):723-730. Doi: 10.1139/010-022.

25. Brann DH, Tsukahara T, Weinreb C, et al. Non-neural expression of SARS-CoV-2 entry genes in the olfactory epithelium suggests mechanisms underlying anosmia in COVID-19 patients. bioRxiv 2020.03.25.009084 [Internet] [cited 2020 Apr 2]. Available from: https://www.biorxiv.org/content/10.1101/2020.03.25.009084v4 Doi: 10.1101/2020.03.25.009084.

26. Wheatland R. Molecular mimicry of ACTH in SARS -implications for corticosteroid treatment and prophylaxis. Med Hypotheses. 2004;63(5):855-862. Doi: 10.1016/j.mehy.2004.04.009.

27. Xu J, Zhao S, Teng T, et al. Systematic comparison of two animalto-human transmitted human coronaviruses: SARS-CoV-2 and SARS-CoV. Viruses. 2020;12(2):244. Doi: 10.3390/v12020244
28. Zha L, Li S, Pan L, et al. Corticosteroid treatment of patients with coronavirus disease 2019 (COVID-19). Med J Aust. 2020;212(9):416-420. Doi: 10.5694/mja2.50577.

29. Трошина Е.А., Никонова Т.В., Свитич О.А. Аутоиммунный полигландулярный синдром взрослых. / Под ред. И.И. Дедова, Е.А. Трошиной. - М.: ГЭОТАР-Медиа; 2019. - 264 с. [Troshina EA, Nikonova TV, Svitich OA. Autoimmunnyi poliglandulyarnyi sindrom vzroslykh. Ed by I.I. Dedov, E.A. Troshina. Moscow: GEOTAR-Media; 2019. 264 p. (In Russ.)]

30. Hamed Azzam S, Kang S, Salvi M, et al. Tocilizumab for thyroid eye disease. Cochrane Database Syst Rev. 2018;11(11):CD012984. Doi: 10.1002/14651858.CD012984.pub2.

31. Imblum BA, Baloch ZW, Fraker D, et al. Pembrolizumabinduced thyroiditis. Endocr Pathol. 2019;30(2):163-167. Doi: 10.1007/s12022-019-9579-2.

32. Трошина Е.А., Сенюшкина Е.С. Вклад центральных регуляторов иммунного ответа в развитие заболеваний щитовидной железы // Проблемы эндокринологии. - 2019. — Т.65. — №6. — C. 458-465. [Troshina EA, Senyushkina ES. The value of central regulators of the immune response in the development of autoimmune thyroid diseases. Problemy endokrinologii. 2019;65(6):458-465. (In Russ.)]. Doi: 10.14341/probl10304.

\section{ИНФОРМАЦИЯ ОБ АВТОРАХ [AUTHORS INFO]}

*Сенюшкина Евгения Семеновна, научный сотрудник [Evgeniya S. Senyushkina, MD];

адрес: Россия, 117036, Москва, ул. Дм. Ульянова, д. 11 [address: 11 Dm. Ulyanova street, 117036 Moscow, Russia]; ORCID: https://orcid.org/0000-0001-7960-8315; eLibrary SPIN: 4250-5123; e-mail: EvgeniyaSenyushkina@yandex.ru

Трошина Екатерина Анатольевна, д.м.н., член-корр. РАН, професcop [Ekaterina A. Troshina, MD, PhD, Professor]; ORCID: https://orcid.org/0000-0002-8520-8702; eLibrary SPIN: 8821-8990; e-mail: troshina@inbox.ru

Мельниченко Галина Афанасьевна, д.м.н., профессор, академик PAH [Galina A. Melnichenko, MD, PhD, Professor]; ORCID: http://orcid.org/0000-0002-5634-7877; eLibrary SPIN: 8615-0038; e-mail: teofrast2000@mail.ru

Мокрышева Наталья Георгиевна, д.м.н., профессор [Natalia G. Mokrysheva, MD, PhD, Professor]; ORCID: http://orcid.org/0000-0002-9717-9742; eLibrary SPIN: 5624-3875; e-mail: nm70@mail.ru

\section{ИНФОРМАЦИЯ}

Рукопись получена: 28.05.2020. Одобрена к публикации: 18.06.2020. Опубликована online: 09.07.2020.

\section{ЦИТИРОВАТЬ}

Трошина Е.А., Мельниченко Г.А., Сенюшкина Е.С., Мокрышева Н.Г. Адаптация гипоталамо-гипофизарно-тиреоидной и гипоталамо-гипофизарно-надпочечниковой систем к новому инфекционному заболеванию - COVID-19 в условиях развития COVID-19-пневмонии и/или цитокинового шторма // Клиническая и экспериментальная тиреоидология. 2020. - T. 16. — №1. — C. 21-27. doi: https://doi.org/10.14341/ket12461

\section{CITE THIS ARTICLE}

Troshina EA, Melnichenko GA, Senyushkina ES, Mokrysheva NG. Adaptation of the hypothalamo-pituitary-thyroid and hypothalamo-pituitary-adrenal systems to a new infectious disease - COVID-19 in the development of COVID-19 pneumonia and/or cytokine storm. Clinical and experimental thyroidology. 2020;16(1): 21-27. doi: https://doi.org/10.14341/ket12461 